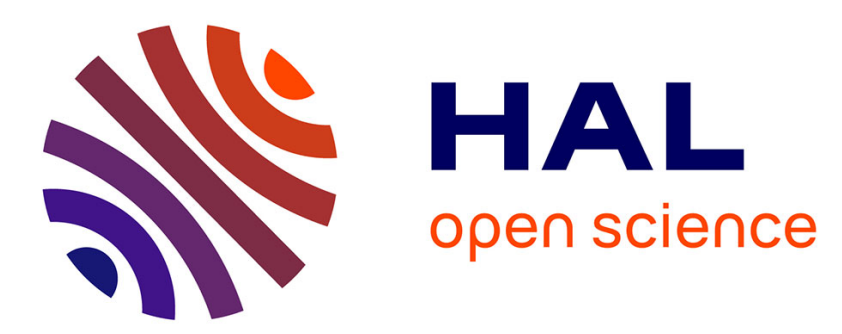

\title{
Adaptive Estimation and Reduction of Noises Affecting a Self-Mixing Interferometric Laser Sensor
}

\author{
Zohaib Khan, Usman Zabit, Olivier D Bernal, Tassadaq Hussain
}

\section{To cite this version:}

Zohaib Khan, Usman Zabit, Olivier D Bernal, Tassadaq Hussain. Adaptive Estimation and Reduction of Noises Affecting a Self-Mixing Interferometric Laser Sensor. IEEE Sensors Journal, 2020, 20 (17), pp.9806-9815. 10.1109/JSEN.2020.2992848 . hal-02959446

\author{
HAL Id: hal-02959446 \\ https://hal.laas.fr/hal-02959446
}

Submitted on 6 Oct 2020

HAL is a multi-disciplinary open access archive for the deposit and dissemination of scientific research documents, whether they are published or not. The documents may come from teaching and research institutions in France or abroad, or from public or private research centers.
L'archive ouverte pluridisciplinaire HAL, est destinée au dépôt et à la diffusion de documents scientifiques de niveau recherche, publiés ou non, émanant des établissements d'enseignement et de recherche français ou étrangers, des laboratoires publics ou privés. 


\title{
Adaptive Estimation and Reduction of Noises Affecting a Self-Mixing Interferometric Laser Sensor
}

\author{
Zohaib A. Khan ${ }^{1}$, Student Member, IEEE, Usman Zabit², Senior Member, IEEE, Olivier D. \\ Bernal $^{3}$ Member, IEEE, and Tassadaq Hussain ${ }^{1}$ \\ ${ }^{1}$ Department of Electrical Engineering, Riphah International University, Islamabad, 44000, Pakistan \\ ${ }^{2}$ Department of Electrical Engineering, National University of Sciences and Technology (NUST), Islamabad, 44000, Pakistan \\ ${ }^{3}$ Universite de Toulouse, LAAS-CNRS, INPT, Toulouse, 31400, France
}

DOI : $10.1109 /$ JSEN.2020.2992848

Abstract - Experimental Self-Mixing (SM) or optical feedback interferometric signals are usually affected by additive white Gaussian noise (AWGN) and impulsive noise. Depending on SM sensing set-up, these noises can significantly reduce the signal to noise ratio (SNR) of SM signals which in turn affects the measurement performance of signal processing algorithms employed for metric information retrieval. In this paper, adaptive line enhancement (ALE) technique is proposed to remove AWGN and impulsive noise from SM signals. Specifically, a recursive least squares (RLS) based ALE algorithm has been designed and the results have been compared with established methods such as high-order digital low-pass filtering and discrete wavelet transform. The comparison indicates better precision in case of use of RLS-ALE even when significant variations occur in the operating optical feedback regime and remote target velocity as well as in presence of speckle. The proposed algorithm can also estimate the SNR of SM signals belonging to weak-, moderate-, and strong-optical feedback regime with SNR ranging from $0 \mathrm{~dB}$ to $40 \mathrm{~dB}$, with a mean absolute error of $1.35 \mathrm{~dB}$ and a $1.09 \mathrm{~dB}$ precision. Statistical analysis of noise recovered from different experimental SM signals attests the Gaussian- and impulsive-nature of noise. Thus, the proposed method also enables a simple and reliable quantitative analysis and comparison of different laser diode based SM laser sensors operating under variable optical conditions.

Index Terms - adaptive filter, noise estimation, optical feedback, self-mixing interferometry, SNR, vibration measurement.

\section{Introduction}

$\mathrm{S}$ elf-mixing interferometry (SMI) or optical feedback interferometry [1]-[3] is being actively researched for velocity [4], displacement [5], distance [6], vibration [7], flow [8], profilometry [9], range-finding [10], temperature [11], and biomedical applications [12], [13] due to the compact, selfaligned, and low-cost nature of the SM instrument.

The metric performance of SMI sensors for different abovementioned applications is highly dependent on the signal to noise ratio of SMI signal $S N R_{S M}$. The experimental SMI signal is invariably contaminated with noises originating within photonic and electronic circuitry while SMI signal's optical feedback (OF) regime, represented by OF coupling parameter $C$ [1], depends on optical path. Commonly, two types of noise exist in SMI signals: impulsive noise, and additive white Gaussian noise [14]. As stated in [15], phase noise in SMI is generated by both the frequency fluctuation of the laser and the target distance fluctuation. Near the SMI discontinuities (for moderate- and strong- OF regime), this phase noise can result in the presence of "impulsive noise" or "fast switching". These noises can severely degrade SMI based measurements, e.g. by causing 1) false detection of SMI fringes and 2) incorrect normalization resulting in unwrapping errors [16]-[19].
$S N R_{S M}$ and bandwidth of SMI signal $b w_{S M}$ are both also dependent on the amount of OF [20]. The signals with low $C$ usually have low $S N R_{S M}$ and smaller $b w_{S M}$, and a significant portion of signal may be buried in noise while the SMI signals corresponding to high OF usually have high $S N R_{S M}$ and larger $b w_{S M}$. So, the detection of SMI signal's features and subsequent processing is easier in such SMI signals having $1<$ $C<4.6$ [16]-[18].

The presence of noise in SMI system is a serious challenge for its sensing and measurement applications. So, in order to retrieve metric information from SMI signal with high accuracy, pre-processing of SMI signal is required to remove noise without distorting the actual SMI signal. Many signal processing techniques exist for noise reduction. The most common technique is digital filtering but the design of digital filters is challenging for wide-band applications as key parameters of digital filters need to be changed if the characteristics of information signal (i.e. SMI signal corresponding to remote target motion) or those of noise change over time. As spectral properties of SMI signal vary as a function of target velocity $v_{t}(t)$ and $\mathrm{OF}$, so digital filters do not provide robust noise removal [20].

Wavelet transform has also been used for the reduction of noise in SMI signal successfully [21]. Wavelet based filter effectively reduces the noise but is limited by the problem of 


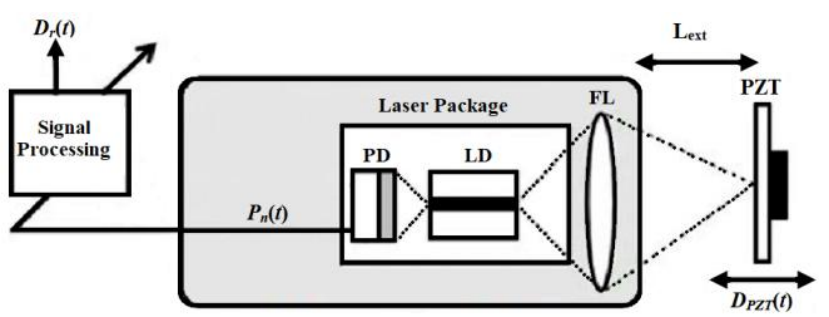

Fig. 1. Block diagram of the adaptive noise estimation and reduction model using RLS-ALE: photodiode (PD), laser diode (LD), focusing lens (FL), and piezoelectric transducer (PZT).

selection of wavelet decomposition level which needs to be varied as a function of operating OF regime and noise power.

For SMI signals affected by impulsive noise, median- and Kaiser-filtering have been reported in [20]. The impulsive noise is greatly reduced by the median filter, but some residual sparkles are left at fringe-level. Kaiser filter also removes the noise effectively, but the peak values of signal are increased thereby distorting the SMI signal.

Outlier detection and myriad filter have also been proposed for removal of SMI impulsive noise [14], [22]. The outlier detection method worked well but only for SMI signal under moderate and strong regimes. The working of myriad filter depends on two key parameters i.e. linear parameter and filter window length. It requires different calibration of both parameters for removal of transient- or additive-noise.

A solution to the above-mentioned problems is adaptive filtering, which has been widely used in environments where power and bandwidth of noise varies over time. As opposed to fixed cut-off frequency based filtering, an adaptive filter adapts its frequency response (e.g. its pass-band and stop-band characteristics) by modifying the filter coefficients or weights. Adaptive filters have been used for noise reduction in communication, speech, radar, and biomedical signals [23].

With respect to SMI, active noise cancellation model (ANC) of adaptive filtering for noise reduction has been proposed in [24]. Least mean squares (LMS) algorithm of adaptive filters was used to automatically adjust the filter weights. ANC effectively reduced the noise from SMI signal, but it required reference noise signal which was derived from the driving source of LD [24]. Use of ANC model has its own limitations as extraction of reference noise signal becomes difficult in situations where noise power is low and may not be correctly detected with a sensor [24].

Therefore, in order to resolve the problems discussed above, in this paper, a single-input adaptive model is presented which can effectively reduce the noises affecting SMI signals by automatically adjusting the filter parameters while not requiring any reference noise signal. This is achieved by using adaptive linear enhancer (ALE) model of adaptive filter. ALE has effectively reduced the noise from SMI signals under large variation in both OF coupling regime as well as remote target velocity conditions. We have used the recursive least squares (RLS) algorithm for updating the ALE. The results of proposed RLS-ALE model have been compared with standard digital low pass filter and wavelet transform based processing showing better performance of RLS-ALE such that the information signal is effectively extracted from noisy SMI signals with high precision.

In addition, RLS-ALE has also been used for simple and direct $S N R_{S M}$ estimation. Usually, $S N R_{S M}$ of SM velocimeter signal is estimated by using the FFT (Fast Fourier Transform) [25], [26] to determine the SM signal spectrum which is then used to find the difference between Doppler signal's peak value and noise-floor level. For displacement/vibration sensing, this approach may not be appropriate as the SM signal's spectrum spreads over multiple harmonics of each of target motion's spectral components. Another possible approach for $S N R_{S M}$ estimation is the use of SMI signal's phase noise, based on variance of fringe discontinuity instant which can be measured by locking the oscilloscope to fringe discontinuity instant [15]. Classical formulation of $S N R_{S M}$, both for photodiode based SMI signal acquisition (PD-SM) or laser diode's terminal voltage based SMI signal acquisition (LV-SM) has been presented in [27]. However, as can be seen in the following section, such $S N R_{S M}$ estimation may not be so straightforward for experimental SM systems because it requires the knowledge of parameters of laser diode, photodiode as well as external optical path.

In this paper, to the best of our knowledge, we present for the first time the use of RLS-ALE for direct and simple $S N R_{S M}$ estimation as well. RLS-ALE enables $S N R_{S M}$ estimation without prior knowledge of SMI-, optoelectronic-, or optical path based-parameters or of noise characteristics. Using the established SMI behavioral model and additive white noise, proposed RLS-ALE has estimated $S N R_{S M}$ with a mean absolute error of $1.35 \mathrm{~dB}$ with a precision of $1.09 \mathrm{~dB}$ over $S N R_{S M}$ range of $0 \mathrm{~dB}$ to $40 \mathrm{~dB}$ whereas $\mathrm{OF}$ strength varied from weak- to strong-feedback regime under simulated conditions. When used on noisy experimental SM signals, the proposed method enabled direct recovery of noise content whose statistical analysis attests the Gaussian and impulsive nature of corresponding noises (presented in section $I V-C$ ).

A schematic block diagram of the proposed adaptive RLSALE based SM sensor is shown in Fig. 1. The SM sensor setup includes laser diode (LD), photodiode (PD), focusing lens (FL) and piezoelectric transducer (PZT) which acts as a vibrating target as well as reference sensor providing $D_{P Z T}(t)$.

The paper is organized as follows. A brief introduction to SM interferometry is provided in section II. Then, the signal processing of the adaptive SM sensor is elaborated in section III. The simulated and experimental results are given in section IV, followed by Discussion and Conclusion.

\section{SELF-MIXING INTERFEROMETRY}

SMI phenomenon occurs in a laser when a small part of the laser light is reflected back by a target having displacement $D(t)$ and is fed back into the internal laser cavity. As a result, the emitted- and reflected-light interfere with each other. This causes variation in the laser output power $P(t)$ given as [1]:

$$
P(t)=P_{0}\left[1+m \cdot \cos \left(\phi_{F}(t)\right)\right]
$$




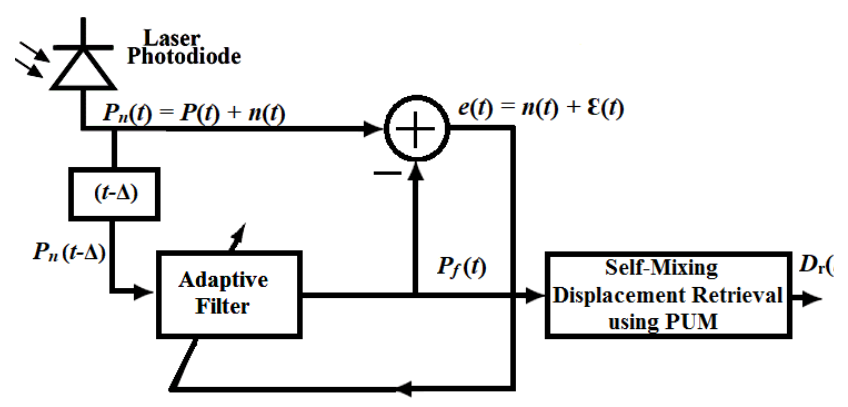

Fig. 2. Signal processing for the adaptive RLS-ALE based SM sensor: PUM (Phase Unwrapping Method) [32] is used to quantify the performance of proposed filter for displacement sensing.

where $P_{0}$ is the emitted optical power without laser feedback, $m$ is the modulation index and $\phi_{F}(t)$ is the laser output phase in the presence of feedback, given by:

$$
\phi_{F}(t)=2 \pi \frac{D(t)}{\lambda_{F}(t) / 2}
$$

Under optical feedback, $\phi_{F}(t)$ is determined by the wellestablished Lang-Kobayashi model [2], given as

$$
\phi_{0}(t)-\phi_{F}(t)-C \sin \left[\phi_{F}(t)+\arctan (\alpha)\right]=0
$$

where $\phi_{0}(t)$ is the laser output phase without the feedback, found by replacing $\lambda_{F}(t)$ with $\lambda$ in (2), where $\lambda$ is the LD emission wavelength in absence of feedback. $\alpha$ is the linewidth enhancement factor [27].

$C$ parameter is a fundamental SMI parameter and is used to specify the SMI operating regime [29]-[31]. $C<1$ characterizes weak OF regime, and the laser output power signal varies in a quasi-sinusoidal manner. When $C>1$ then the SMI fringe shape becomes sharper, resulting in a sawtooth like signal. As previously mentioned, spectral properties of an SMI signal (e.g. $b w_{S M}$ ) are a function of both OF coupling as well as $v_{t}(t)$ (which is itself a function of target vibration's frequency and amplitude). For a given $v_{t}(t)$, lower (higher) OF coupling results in lower (higher) $b w_{S M}$ [20].

$S N R_{S M}$ of photodiode based SMI signal acquisition (as schematized in Fig. 1) has been formulated in [27] as

$$
S N R=2 I_{p h 0}\left(\frac{t_{1}^{2}}{r_{1}}\right)^{2}\left(\frac{A}{e B}\right)\left(2 \gamma L+\ln R_{1} R_{2}\right)^{-2}
$$

where $R_{1}=r_{1}{ }^{2}, R_{2}=r_{2}^{2}$ ( $r_{1}$ and $r_{2}$ are the field reflections at the front- and back-mirror of LD), $t_{1}$ is the field transmission at the front mirror, $L$ is the cavity length, $I_{p h 0}$ is the photo-detected current, $B$ is the bandwidth of observation, $e$ is the electron charge, $\gamma$ is the power gain per unit length of the active medium, and $A^{1 / 2}$ is the field attenuation suffered in propagation, including the diffusion at the target surface [27].

\section{Signal PROCESSING}

The signal processing of adaptive RLS-ALE SM sensor (see Fig. 2) can be grouped into two major parts, detailed below.

\section{A. Self-mixing Interferometric Signal:}

The photodiode (PD) inside the laser diode (LD) package (see Fig. 1) is typically used to acquire the SMI signal $P(t)$.
As previously mentioned, spectral properties of an SMI signal (e.g. $b w_{s m}$ ) are a function of both OF coupling as well as $v_{t}(t)$ (which is itself a function of target vibration's frequency and amplitude). This can be verified from (1-3) indicating that higher rate of change of $D(t)$ leads to higher rate of change of $\phi_{0}(t), \phi_{F}(t)$, and $P(t)$ in the same order._The processing of this interferometric signal leads to the reconstruction of corresponding displacement or vibration signal. To achieve accurate vibration or displacement sensing, a high-quality $P(t)$ signal is required. This paper focuses on recovering high quality $P(t)$ by using the proposed RLS-ALE model shown in Fig. 2. The acquired noisy SMI signal is denoted as $P_{n}(t)=$ $P(t)+n(t)$ where $n(t)$ denotes noise.

\section{B. Adaptive Filtering for noise reduction:}

ANC is one of the basic and mostly commonly used models for noise reduction. However, it requires a reference noise signal, which is usually not available in real-world scenarios [33], [34]. A solution to this limitation is ALE which does not require reference noise signal [23].

In the proposed ALE model, noisy SMI signal is used as the input signal as well as the reference signal (which is the same input signal but delayed by the factor $\Delta$ as shown in Fig. 2 . The delayed version of noisy SMI signal $P_{n}(t-\Delta)$ is fed into the adaptive filter (see Fig. 2) and filtered signal $P_{f}(t)$ is obtained at the output, which is further processed to retrieve the displacement signal. In this paper, PUM (phase unwrapping method) [32] is used for the retrieval of displacement signal represented by $D_{r}(t)$.

\section{1) Recursive Least Squares (RLS) Algorithm}

RLS adaptive algorithm recursively updates the weights (or coefficients) of the adaptive filter so that the weighted linear least squares cost function is minimized with respect to the input signal. The RLS algorithm is known for its excellent performance while working in time varying environment but at the cost of an increased computational complexity and some stability constraints [23]. In this work, RLS-ALE requires two inputs: input noisy signal denoted as $X(n)$ and its delayed version denoted as $X(n-\Delta)$ which acts as the reference noise signal. The error signal in this case is the difference between input signal and weighted reference signal given by [23]:

$$
e(n)=X(n)-w^{T} X(n-\Delta)
$$

In our case the input signal becomes $P_{n}(t)$ as shown in the Fig. 2. The output of adaptive filter is the weighted signal which is the filtered signal denoted by $P_{f}(t)$. RLS filter coefficients are updated by [23]

$$
w(n)=w(n-1)+k(n) e(n)
$$

where $k(n)$ is the filter gain given by [23]

$$
k(n)=\frac{\Lambda^{-1} \emptyset_{y}(n-1) X(n-\Delta)}{1+\Lambda^{-1} X^{T}(n-\Delta) \emptyset_{y}(n-1) X(n-\Delta)}
$$

where $\Lambda$ is the adaptation factor, and $\emptyset_{y}$ is the correlation matrix, and is recursively updated by [23]

$$
\emptyset_{y}(n)=\Lambda^{-1} \emptyset_{y}(n-1)-\Lambda^{-1} k(n) X^{T}(n-\Delta) \emptyset_{y}(n-1)
$$

The initial value of $\emptyset_{y}(n)$ is equal to the product of identity matrix $\boldsymbol{I}$ and regularization parameter $\delta$ given by [23] 


$$
\emptyset_{y}(n)=\delta \boldsymbol{I}
$$

The stability of RLS algorithm can be controlled by $\delta$. A small positive constant number is assigned to $\delta$ for the reduction of noise from signals [23].

\section{RESULTS}

Simulated and experimental SMI signals have been tested using RLS-ALE adaptive noise reduction model. Same signals have been tested by using standard LPF and DWT, and the results are compared, as detailed below.

\section{A. Results of Simulated SMI Signals}

To establish fair comparison between ALE-RLS, LPF, and DWT based filtering, different SMI specific parameters such as $C, \alpha$, and target vibration frequency $f_{P Z T}$ were used to cover the usual parameter range of interest. Then, filter-specific parameters of LPF (type of filter, order of filter $N_{L P F}$, and cutoff frequency $f_{c}$ ), and of DWT (type of wavelet, order of filter $N_{D W T}$, decomposition-level of filter $L_{D W T}$ ) were varied until their corresponding error performance compared favorably with the deployed RLS-ALE based filter. Error performance of these three filtering schemes (after this optimization of LPF and DWT) is discussed below. Note that RLS-ALE filter order $N_{A L E}$ is kept constant to 150 while $\Delta$ is set to 5 in all the cases. $\left(N_{A L E}=150\right.$ was chosen as the optimized value after multiple simulations as lower $N_{A L E}$ gave higher measurement error while higher $N_{A L E}$ did not improve error performance.)

Firstly, it was simulated to test if the RLS-ALE model can self-adaptively remove the noise in an SMI signal belonging to weak OF regime $(C=0.9)$ with $\lambda=0.785 \mu \mathrm{m}$ and $\alpha=5$, simulated by employing the SM behavioral model [35]. This behavioral model enables generating the SM signal $P(t)=$ $\cos \left(\phi_{F}(t)\right)$ corresponding to given $D(t)$ by solving the excess phase equation (3) as a function of $C, \alpha$, and $\phi_{0}(t)=\frac{4 \pi}{\lambda} D(t)$. Target vibration $f_{P Z T}$ was set to $100 \mathrm{~Hz}$ with $A_{\mathrm{p}-\mathrm{p}}=4.1 \mu \mathrm{m}$ and a sampling frequency of $f_{S}=100 \mathrm{kHz}$ while $S N R_{S M}$ was $12.2 \mathrm{~dB}$ (see Fig. 3). $N_{L P F}$ was set to 5000 while $f_{c}$ was set to $1 \mathrm{kHz}$, as per previously described optimization of LPF parameters required to achieve the optimum results. Similarly, Daubechies wavelet with $N_{D W T}=4$ was chosen for reduction of noise (as it has similarity with the shape of SMI signal fringe [21]) while the optimum result of DWT is obtained by setting $L_{D W T}$ to 3.

To compare the performance of RLS-ALE model with LPF and DWT based filtering, the filtered SMI signals were processed by PUM [36] and the retrieved $D_{r}(t) 1$ was compared with the reference displacement signal $D_{\mathrm{PZT}}(t)$. The displacement signals recovered by using RLS-ALE, LPF and DWT shown in dotted yellow, green, and red respectively, match well with the reference $D_{\mathrm{PZT}}(t)$ signal shown in blue (see Fig. 3(f)). The error signal between reference and retrieved-displacement signal by RLS-ALE, LPF and DWT is shown in Fig. 3(g), with RMS error $\epsilon_{A L E}, \epsilon_{L P F}$, and $\epsilon_{D W T}$ of $19.1 \mathrm{~nm}, 38.3 \mathrm{~nm}$, and $21.3 \mathrm{~nm}$, respectively.

Secondly, one of the operating (target motion) parameters (in this case $f_{P Z T}$ ) was changed while keeping all the other parameters the same. Fig. 4 represents the case where $f_{P Z T}=$
$10 \mathrm{~Hz}$ while keeping all the other parameters the same. RLSALE has removed the noise effectively with $\epsilon_{A L E}=28.5 \mathrm{~nm}$. However, LPF and DWT performance degrades with $\epsilon_{L P F}=146 \mathrm{~nm}$ and $\epsilon_{D W T}=1952 \mathrm{~nm}$. Note that the threshold used for correct fringe detection (FD) within PUM was optimized to enable as good FD as possible for all three algorithms. However, ineffective noise reduction by LPF and DWT resulted in false FD (one incorrect fringe in case of LPF and eight incorrect fringes in case of DWT) which caused corresponding increase in measurement error.

Thirdly, Fig. 5 represents the case where $f_{P Z T}=1 \mathrm{kHz}$ with $A_{\mathrm{p}-\mathrm{p}}=4.1 \mu \mathrm{m}$ and the corresponding simulated SMI signal has the same parameters of $C=0.9, \lambda=0.785 \mu \mathrm{m}$, and $\alpha=5$ as shown in Fig. 5 (a). The sampling frequency in this case is set to $f_{S}=1 \mathrm{MHz}$. The RLS-ALE model has effectively removed the noise without significantly distorting the shape of the SMI signal, while the LPF and DWT have also removed the noise but the shape of SMI signal is significantly distorted (indicating that significant spectral content of SMI signal has also been removed by the LPF and DWT). It is reiterated that the filter-specific parameters of LPF and DWT are kept the same as those of the first case of $f_{P Z T}=100 \mathrm{~Hz}$ (for which optimum error performance results were obtained). The displacement signal recovered from RLS-ALE matches well with $D_{P Z T}(t)$, while the displacement signals recovered by DWT and LPF deviate from $D_{P Z T}(t)$, as quantified by the RMS error of $\epsilon_{A L E}=20.1 \mathrm{~nm} \epsilon_{L P F}=2859 \mathrm{~nm}$ and $\epsilon_{D W T}=$ $9861 \mathrm{~nm}$. The RMS error shows that the performance of LPF and DWT drastically falls because of wrongly detected fringes while RLS-ALE has maintained its performance.

Fourthly, it was simulated to test if the RLS-ALE, LPF and DWT can provide acceptable correction if $C$ changes (e.g. if it increases from low- to strong-OF regime) while keeping $f_{P Z T}$ and all the other parameters the same.

Fig. 6 presents the case of $f_{P Z T}=100 \mathrm{~Hz}$ and $C=4.9$, and $\alpha=5$. Again, RLS-ALE has removed the noise effectively without distorting the shape of SM signal with $\epsilon_{A L E}=15.1$ $\mathrm{nm}$. The DWT has also removed the noise to major extend, without distorting the shape of SMI fringes, yet, presence of noise can be seen within filtered signal while the LPF has distorted the shape of SMI fringes, as quantified by $\epsilon_{L P F}=$ $3507 \mathrm{~nm}$ and $\epsilon_{D W T}=7206 \mathrm{~nm}$ due to some missed fringes.

Thus, it is observed that change in $C$ alone (while keeping all other parameters the same) can degrade the performance of both LPF and DWT while RLS-ALE maintains its performance due to its adaptive nature.

Various other simulations were also performed by changing the value of $C$ and $S N R_{S M}$ for $f_{P Z T}=50 \mathrm{~Hz}$ and $f_{P Z T}=$ $500 \mathrm{~Hz}$ while keeping all other parameters the same. These results are summarized in Fig. 7.

\section{B. SNR Estimation using RLS-ALE}

In most experimental systems, direct and real-time access to noise is not available making it difficult to estimate the SNR of experimental signals. Proposed RLS-ALE solves this problem by not just giving the estimate of information signal (i.e. $P_{f}(t)$ ) but the estimate of noise residue (i.e. $e(t)$ ) as well. 


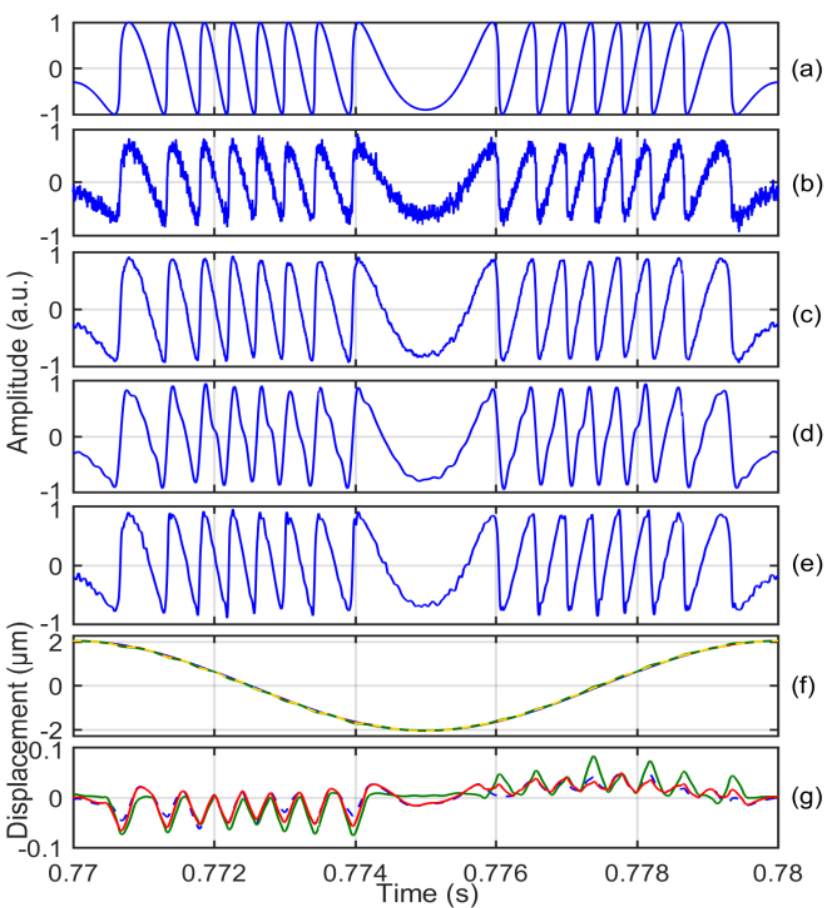

Fig. 3. Simulation for $f_{P Z T}=100 \mathrm{~Hz}$ : (a) SMI signal with $C=0.9$ and $\alpha=5$, (b) Noisy SM signal with SNR = $12.2 \mathrm{~dB}$, (c) SM signal recovered with RLS-ALE, (d) SM signal recovered with LPF, (e) SM signal recovered with DWT, (f) Displacement $D_{r}(t)$ retrieved by PUM, (blue (reference target displacement $D_{\text {PZT }}(t)$ ), yellow dotted (RLS-ALE), red (DWT) and green (LPF), $(\mathrm{g})$ Error e $(t)=D_{\mathrm{PZT}}(t)-$ $D_{r}(t)$ [RLS-ALE(dotted blue), LPF (green), DWT (red)].

These estimates can then be used to estimate $S N R_{S M}$ as per

$$
S N R=10 \log _{10} \frac{(\text { Signal Power })}{(\text { Noise Power })}
$$

Multiple simulations were conducted by varying OF strength from weak- to moderate- to strong-OF regime while $S N R_{S M}$ was varied from approximately $0 \mathrm{~dB}$ to $40 \mathrm{~dB}$ (see Table I). Comparison with actual $S N R_{S M}$ values shows that RLS-ALE has estimated $S N R_{S M}$ with absolute mean error and standard deviation of $1.35 \mathrm{~dB}$ and $1.09 \mathrm{~dB}$, respectively. This thus indicates the use of RLS-ALE as a reliable yet simple estimator of SNR for different SM sensing systems.

\section{Results of Experimental SMI Signals}

The experimental set-up deployed for the validation of RLS-ALE sensing model has been schematized in Fig. 1. The laser diode used for SM sensing is a Sanyo ${ }^{\circledR}$ DL7140 with $\lambda=785 \mathrm{~nm}$ and output power of $50 \mathrm{~mW}$. A commercial PZT actuator from Physik Instrumente (P753.2CD) served as target. This device also has a built-in capacitive feedback sensor with $2 \mathrm{~nm}$ resolution that served as a reference sensor for the PZT displacement $D_{P Z T}(t)$. It thus enables error measurements between the recovered displacement signal $D_{r}(t)$ and the reference motion $D_{P Z T}(t)$.

So, firstly, using $f_{P Z T}=40 \mathrm{~Hz}$, a noisy SMI signal is acquired with (PUM based) estimated $C$ value of $\hat{C}=2.3$ and estimated $S N R_{S M}$ of $4.83 \mathrm{~dB}$ (see Fig. 8(a)). In spite of poor SNR, the ALE with $N_{A L E}=150$ has eliminated noise in a very effective way without distorting the SMI signal shape significantly.

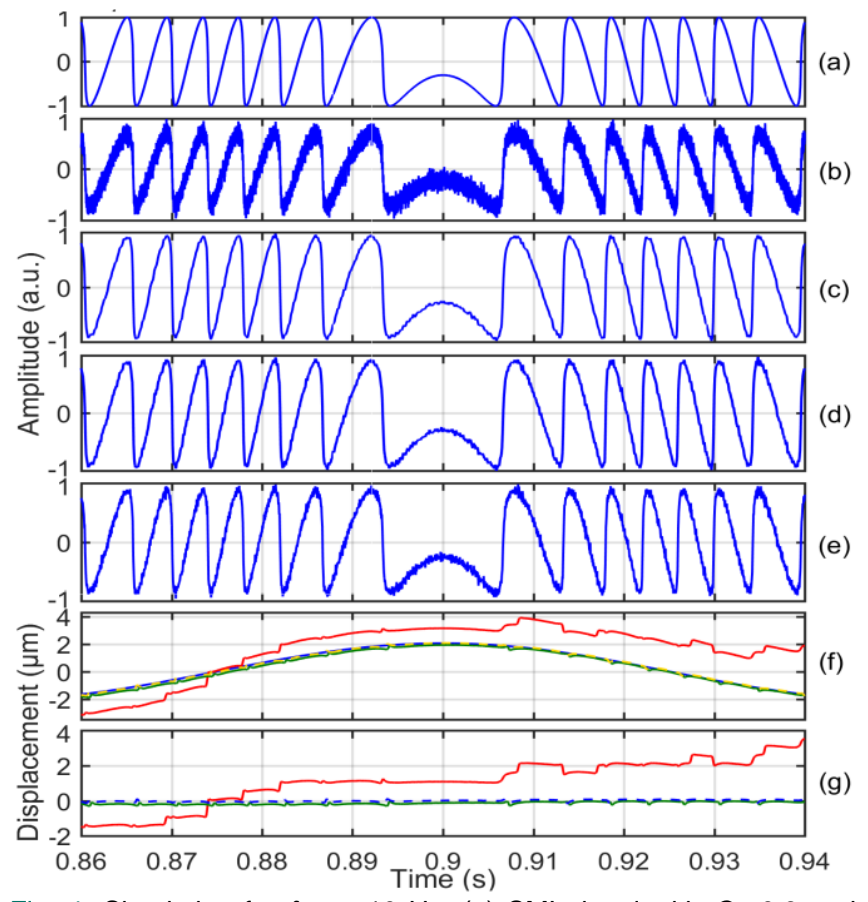

Fig. 4. Simulation for $f_{P Z T}=10 \mathrm{~Hz}$ : (a) SMI signal with $C=0.9$ and $\alpha=5$, (b) Noisy SM signal with $S N R=12.1 \mathrm{~dB}$, (c) $\mathrm{SM}$ signal recovered with RLS-ALE, (d) LPF, (e) DWT, (f) $D_{r}(t)$ retrieved by PUM, blue $\left(D_{\text {PZT }}(t)\right)$, yellow dotted (RLS-ALE), red (DWT) and green (LPF), (g) Error [RLS-ALE(dotted blue), LPF (green), DWT (red)].

To achieve optimum results, $N_{L P F}, f_{c}$ and $L_{D W T}$ were set to $5000,2 \mathrm{kHz}$, and 2, respectively. Thus, LPF and DWT also removed the noise successfully without distorting shape of SMI signal. The retrieved displacement signals are shown in the Fig. 8(e) with the rms errors of $\epsilon_{A L E}=32.1 \mathrm{~nm}, \epsilon_{L P F}=$ $72.3 \mathrm{~nm}$ and $\epsilon_{D W T}=245 \mathrm{~nm}$.

Then, the same experimental SMI signal was processed by using the filter parameters of LPF and DWT chosen for processing simulated SMI signals (i.e. $f_{c}=1 \mathrm{kHz}$ and $L_{D W T}$ $=3$ ). Thus, one key parameter was changed in LPF and DWT to observe the corresponding effect on error performance.

Expectedly, the performance of LPF and DWT is reduced if a key parameter $\left(\mathrm{C}, f_{P Z T}, \ldots\right)$ is changed with RMS errors of $\epsilon_{L P F}=1787 \mathrm{~nm}$ and $\epsilon_{D W T}=728 \mathrm{~nm}$ respectively.

Secondly, an experimental SMI signal corresponding to dual-tone target vibration was processed (PZT was vibrating at $52 \mathrm{~Hz}$ and $85 \mathrm{~Hz}$ with $A_{\mathrm{p}-\mathrm{p}}=5 \mu \mathrm{m}$ and $A_{\mathrm{p}-\mathrm{p}}=7 \mu \mathrm{m}$ respectively). The noisy SMI signal had SNR of $9.15 \mathrm{~dB}$. To achieve the optimal result, $N_{L P F}, f_{C}$ and $L_{D W T}$ was adjusted to $5000,2.5 \mathrm{kHz}$ and 3 , respectively. The error plots are shown in Fig. 9(e) with $\epsilon_{A L E}=24.2 \mathrm{~nm}, \epsilon_{L P F}=34.1 \mathrm{~nm}$ and $\epsilon_{D W T}=38.6 \mathrm{~nm}$, showing the best performance of RLS-ALE (while always using $N_{A L E}=150$ ). When the same signal was processed by using the LPF and DWT parameters chosen for the first case (i.e. $f_{c}=2 \mathrm{kHz}$ and $L_{D W T}=2$ ), the RMS error values increase sharply to $\epsilon_{L P F}=424 \mathrm{~nm}$ and $\epsilon_{D W T}=741 \mathrm{~nm}$.

The above-mentioned results indicate that RLS-ALE performance remains consistent while the filter parameters of LPF and DWT need to be changed as a function of SM signal characteristics. Interestingly, even after optimization of LPF 
1
0
-1

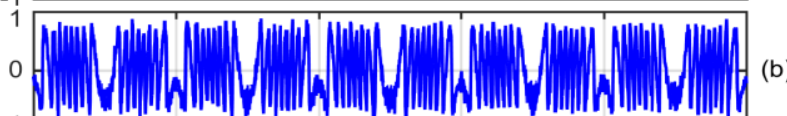

, 1 ,

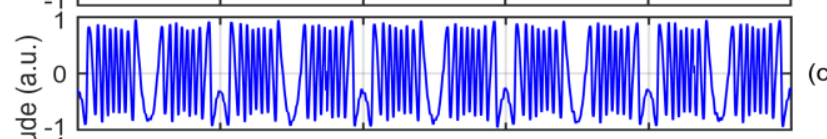

(c)
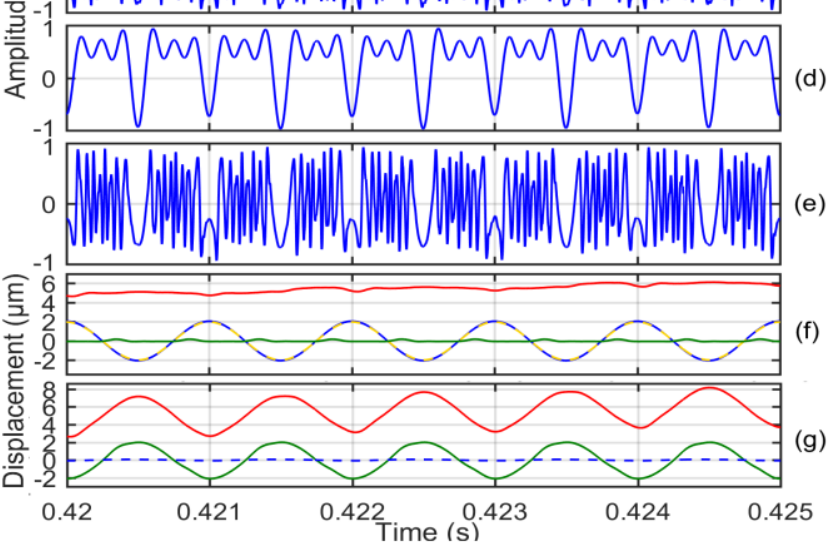

Fig. 5. Simulation for $f_{P Z T}=1 \mathrm{kHz}$ : (a) SMI signal with $C=0.9$ and $\alpha=5$, (b) Noisy SM signal with $S N R=12.2 \mathrm{~dB}$, (c) $\mathrm{SM}$ signal recovered with RLS-ALE, (d) ) LPF, (e) DWT, (f) $D_{r}(t)$ retrieved by PUM, blue $\left(D_{\mathrm{PZT}}(t)\right)$, yellow dotted (RLS-ALE), red (DWT) and green (LPF), (g) Error [RLS-ALE(dotted blue), LPF (green), DWT (red)].

and DWT parameters, performance of RLS-ALE remains better (as seen in Table II which includes the error performance for other experimental SMI signals as well while using optimized LPF and DWT filter parameters).

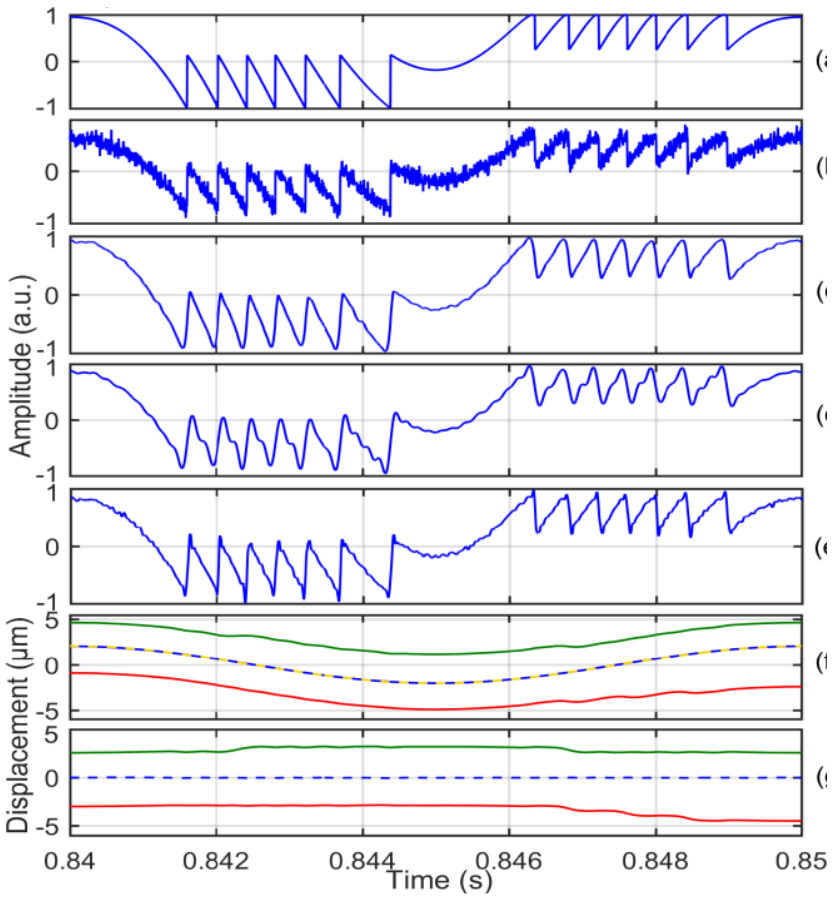

Fig. 6. Simulation for $f_{P Z T}=100 \mathrm{~Hz}$ : (a) SMI signal with $C=4.9$ and $\alpha=5$, (b) Noisy $\mathrm{SM}$ signal with $\mathrm{SNR}=12.1 \mathrm{~dB}$, (c) $\mathrm{SM}$ signal recovered with RLS-ALE, (d) LPF, (e) DWT, (f) $D_{r}(t)$ retrieved by PUM, (blue $\left(D_{\text {PZT }}(t)\right)$, yellow dotted (RLS-ALE), red (DWT) and green (LPF), (g) Error [RLS-ALE(dotted blue), LPF (green), DWT (red)].

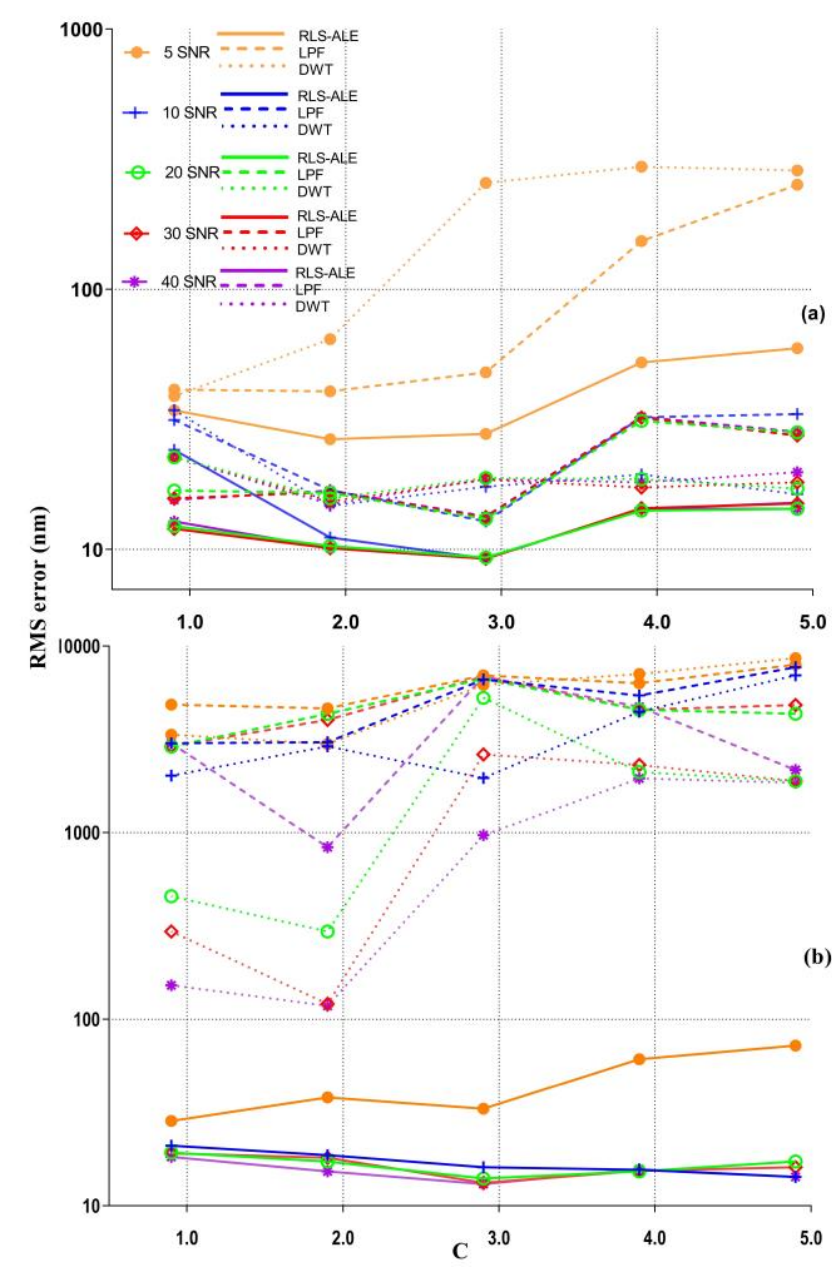

Fig. 7. PUM based RMS error results in displacement measurements of simulated SMI signals for different optical feedback, and SNR: (a) $f_{P Z T}=50 \mathrm{~Hz}$, and (b) $f_{P Z T}=500 \mathrm{~Hz}$.

TABLE I

RLS-ALE BASED SNR ESTIMATION OF SIMULATED SMI SIGNALS FOR DIFFERENT OPTICAL FEEDBACK AND NOISE POWER

\begin{tabular}{|c|c|c|c|c|}
\hline $\begin{array}{l}\text { PZT } \\
(\mathbf{H z})\end{array}$ & $\mathbf{C}$ & $\begin{array}{l}\text { SNR using } \\
\text { (10) }(\text { dB })\end{array}$ & $\begin{array}{c}\text { SNR using } \\
\text { RLS-ALE (dB) }\end{array}$ & $\begin{array}{l}\text { Absolute Error } \\
\text { (dB) }\end{array}$ \\
\hline \multirow{18}{*}{50} & \multirow{6}{*}{0.9} & 0.4 & 2.8 & 2.4 \\
\hline & & 6.01 & 5.06 & 0.95 \\
\hline & & 10.27 & 8.3 & 1.97 \\
\hline & & 20.8 & 21.8 & 1 \\
\hline & & 30.8 & 31.3 & 0.5 \\
\hline & & 40.9 & 41.6 & 0.7 \\
\hline & \multirow{6}{*}{2.9} & 0.50 & 1.47 & 0.97 \\
\hline & & 6.03 & 5.2 & 0.83 \\
\hline & & 10.05 & 9.31 & 0.74 \\
\hline & & 20.07 & 20.01 & 0.06 \\
\hline & & 30.06 & 30.6 & 0.54 \\
\hline & & 40.06 & 40.1 & 0.04 \\
\hline & \multirow{6}{*}{4.9} & 0.3 & 3.1 & 2.8 \\
\hline & & 6.1 & 6.7 & 0.6 \\
\hline & & 10.1 & 11.3 & 1.2 \\
\hline & & 20.04 & 17.9 & 2.14 \\
\hline & & 30.7 & 26.9 & 3.8 \\
\hline & & 40.05 & 43.2 & 3.15 \\
\hline \multicolumn{4}{|c|}{ Mean / Standard deviation of Absolute Error } & $1.35 / 1.09$ \\
\hline
\end{tabular}




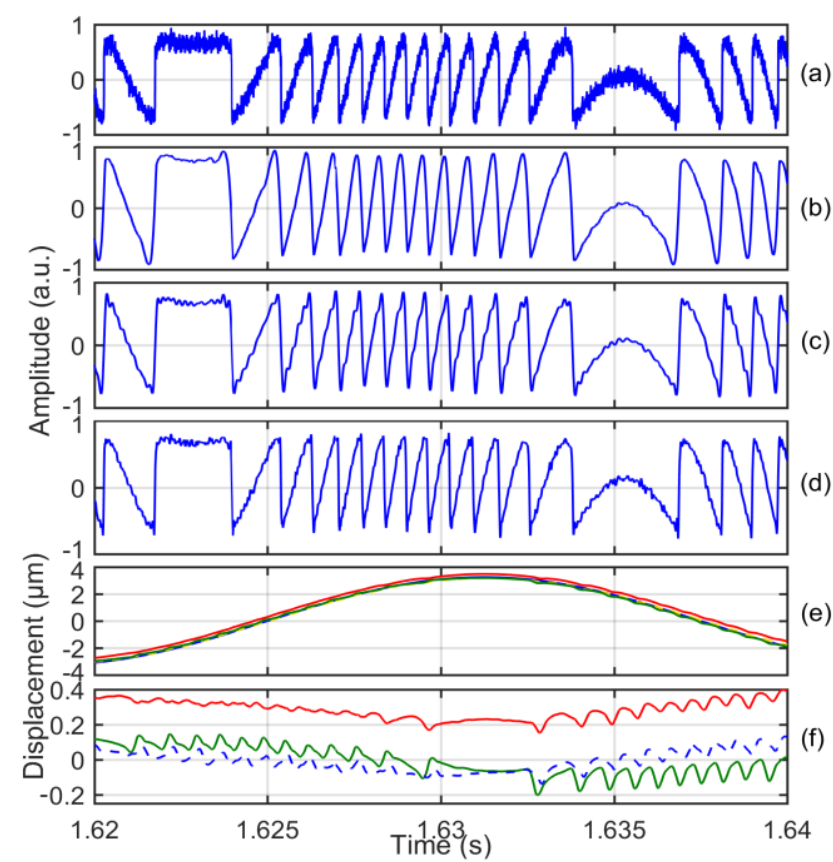

Fig. 8. Experimental signals for $f_{P Z T}=40 \mathrm{~Hz}$ : (a) experimental noisy SMI signal picked up by DL7140, (b) SM signal recovered with RLS-ALE, (c) LPF, (d) DWT, (e) $D_{r}(t)$ retrieved by PUM, (blue $\left(D_{P Z T}(t)\right)$, yellow dotted (RLS-ALE), red (DWT) and green (LPF), (f) Error [RLS-ALE(dotted blue), LPF (green), DWT (red)].

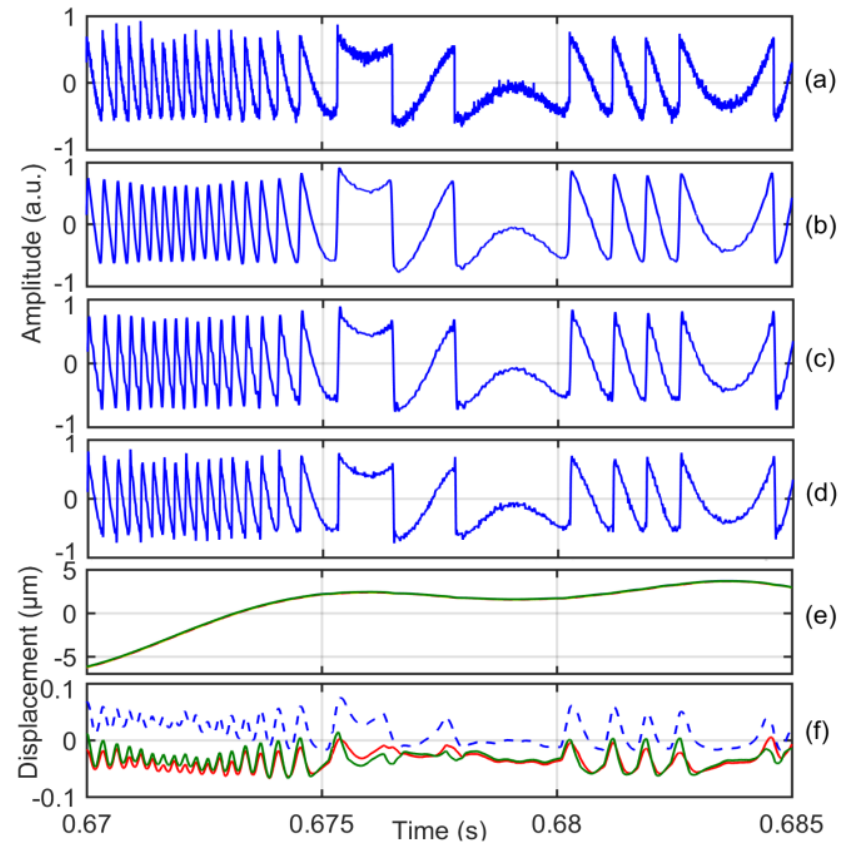

Fig. 9. Experimental signals for $f_{P Z T}=52-85 \mathrm{~Hz}$ : (a) experimental noisy SMI signal picked up by DL7140, (b) SM signal recovered with RLS-ALE, (c) LPF, (d) DWT, (e) $D_{r}(t)$ retrieved by PUM, (blue $\left(D_{P Z T}(t)\right)$, yellow dotted (RLS-ALE), red (DWT) and green (LPF), (f) Error [RLS-ALE(dotted blue), LPF (green), DWT (red)].

\section{1) Impulsive-noise affected SMI signals}

Different impulsive-noise affected SMI signals were also processed proposed RLS-ALE. Due to self-adaptive nature of RLS-ALE, good impulsive noise removal is achieved, as shown in Fig. 10, and Fig. 11. Note that Fig. 10 (a) presents an experimental SMI signal corresponding to vibration at $8 \mathrm{kHz}$ with amplitude $<1 \mu \mathrm{m}$ while Fig. 11 (a) presents another SMI signal corresponding to $170 \mathrm{~Hz}$ vibration with $23 \mu \mathrm{m}$ amplitude. RLS-ALE estimated SNR $_{\mathrm{SM}}$ to be $29.5 \mathrm{~dB}$ and 35.3 $\mathrm{dB}$, respectively.

\section{2) Speckle affected SMI signals}

RLS-ALE was also tested on speckle affected SMI signal to check if it can remove noise in all or specific region of the said signal. The speckle affected SMI signals appear as amplitude modulated signals with variable $C$ caused by the incoherent superposition of signals reflected from the rough target surface [19]. Fig. 12 shows that RLS-ALE has eliminated both impulsive- and white-noise in an effective manner for a speckle affected SMI signal as well.

\section{3) Current modulated SMI signals}

RLS-ALE was used to filter a very noisy experimental SMI signal recovered from a LD current-modulated SMI sensing set-up. Current modulation of the laser diode causes modulation of laser power which acts as a dithering signal [37]. Consequently, SMI signal is embedded within the modulated laser power (shown in Fig. 13 (a)). Recovered SMI signal is presented in Fig. 13 (b) which has been successfully de-noised by RLS-ALE with estimated $S N R_{S M}$ of $2.85 \mathrm{~dB}$.

\section{4) Statistical analysis of experimental noise}

As RLS-ALE enables recovery of noise from the SM signal, so it is possible to perform statistical analysis of noise which is useful in determining the characteristics and distribution of experimental noise of a given SMI sensing set-up.

Fig. 14 (a) presents the statistical distribution of noise recovered from the SMI signal already presented in Fig. 13 (b). It can be clearly seen that this specific experimental noise satisfies the Gaussian noise model with standard deviation $(\sigma)$ of 0.28 and mean $(\mu)$ of 0.00084 .

Likewise, Fig. 14 (b) presents the statistical distribution of impulsive noise recovered from the SMI signal shown in Fig. 11 (a) with $\sigma=0.0439$ and $\mu=-0.00041$.

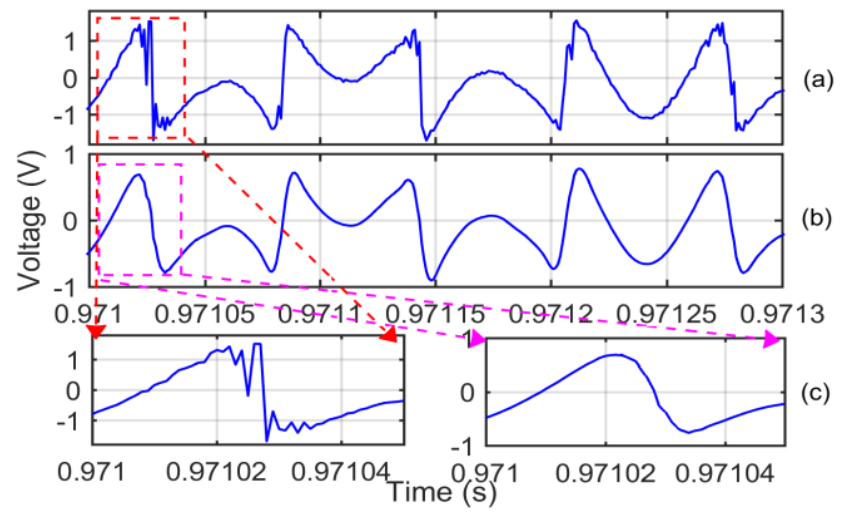

Fig. 10. (a) Experimental SM signal affected by impulsive noise corresponding to vibration at $8 \mathrm{kHz}$ with amplitude $<1 \mu \mathrm{m}$, (b) RLSALE based filtered SM signal, and (c) enlarged view of an affected fringe before and after filtering.

\section{DISCUSSION}

\section{Comparison of RLS-ALE with DWT and LPF}

As seen in previous results, the proposed RLS-ALE scheme is applicable on all major feedback regimes of SMI signal as well as remote-target frequency without requiring any userdriven change in filter-specific parameters. 


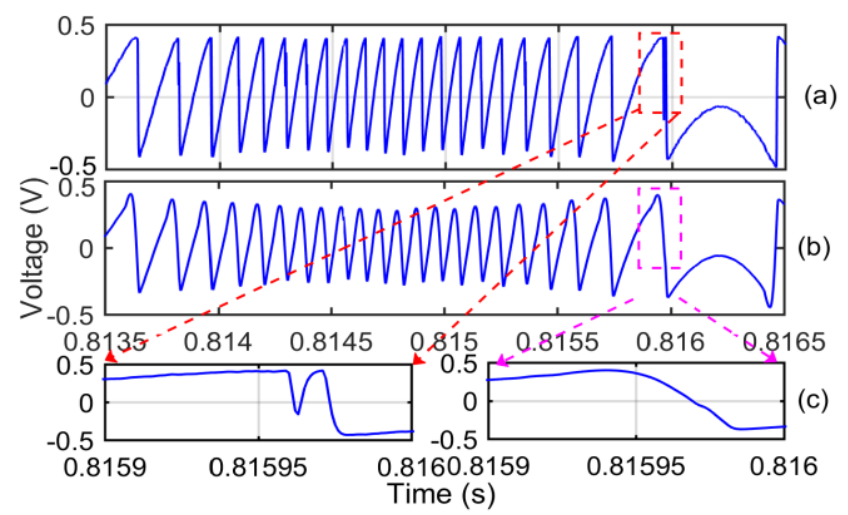

Fig. 11. (a) Experimental SMI signal affected by impulsive noise for multi-micrometric displacement, (b) RLS-ALE based filtered SMI signal, and (c) enlarged view of an affected fringe.

On the other hand, the LPF and DWT based filtering work well only under specific settings of filter-parameters, i.e., change in one of the key parameters can lead to significant degradation in performance. The key advantage of RLS-ALE is that it does not require any extraneous/additional information/input for processing noisy signals. Also, unlike other adaptive configurations, it is a single-input algorithm (i.e., no additional noise estimate/reference is needed).

Another important property of RLS-ALE which makes it superior to LPF or DWT is that it is also applicable on such SMI signals having time-varying characteristics.

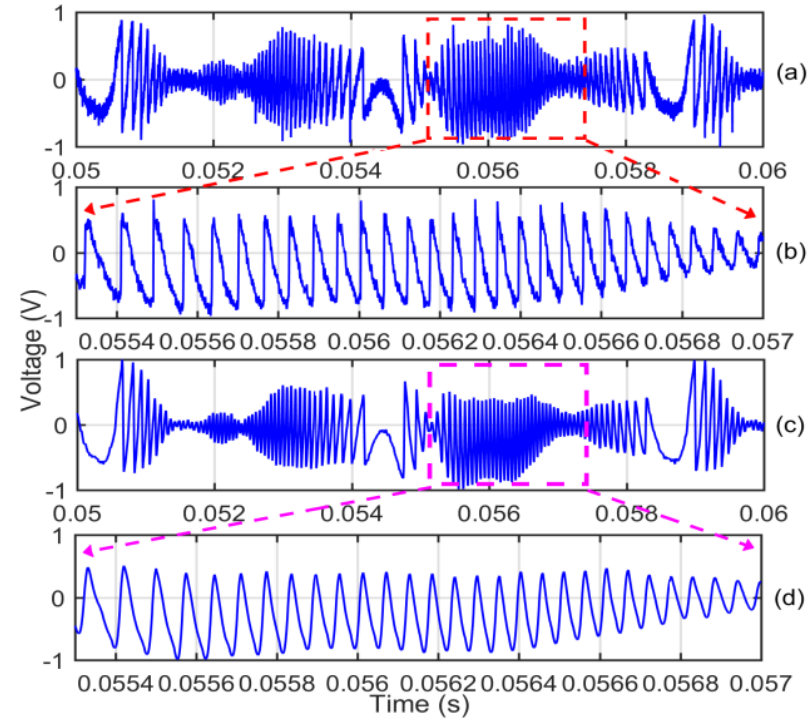

Fig. 12. (a) Experimental speckle affected signal for $f_{P Z T}=110 \mathrm{~Hz}$ : picked up by DL7140, (b) enlarged view of red dotted block of signal in (a), (c) SM signal recovered with RLS-ALE, (d) enlarged view of pink dotted block of signal in (c).

In the speckle affected SMI signals with varying OF coupling, the LPF or the DWT works well for one particular signal segment but fails in other segments, while the RLS-ALE works well in all segments. The reason behind this is the fixed cut-off frequency and decomposition level of LPF and DWT, respectively. These parameters may work well for one OF regime but under-perform for other OF regime while RLSALE adapts itself to characteristics of SMI signal.

The main limitation of the RLS-ALE as compared to LPF and DWT is its far higher computational complexity, as seen in (5-8). RLS-ALE roughly requires $3 N^{2}+11 N$ multiplications [23] as opposed to DWT and LPF which require $2 N$ (per decomposition-level) and $N$ multiplications, respectively; where $N$ denotes the filter-order.

TABLE ॥

PUM BASED RMS ERROR RESULTS IN THE DIPSLACEMENT MEASUREMENT OF EXPERIMENTAL SMI SIGNALS FOR DIFFERENT TARGET VIBRATIONS, OPTICAL FEEDBACK AND SNR

\begin{tabular}{|c|c|c|c|c|c|}
\hline $\begin{array}{l}\text { PZT } \\
(\mathbf{H z})\end{array}$ & $\hat{C}$ & $\begin{array}{l}\text { Estimated } \\
\text { SNR (dB) }\end{array}$ & $\begin{array}{r}\epsilon_{A L E} \\
(\mathbf{n m})\end{array}$ & $\begin{array}{c}\boldsymbol{\epsilon}_{L P F} \\
(\mathbf{n m})\end{array}$ & $\begin{array}{c}\epsilon_{D W T} \\
(\mathrm{~nm})\end{array}$ \\
\hline 40 & 2.3 & 4.83 & 32.1 & 72.3 & 245 \\
\hline $52-85$ & 2.9 & 9.15 & 24.2 & 34.1 & 38.6 \\
\hline 70 & 2.2 & 3.82 & 34 & 64.9 & 43.2 \\
\hline 100 & 2.4 & 8.87 & 22.1 & 43.6 & 36.1 \\
\hline $35-175$ & 2.1 & 3.3 & 27.2 & 39.7 & 42.1 \\
\hline \multicolumn{3}{|c|}{ mean } & 27.9 & 50.9 & 81 \\
\hline
\end{tabular}

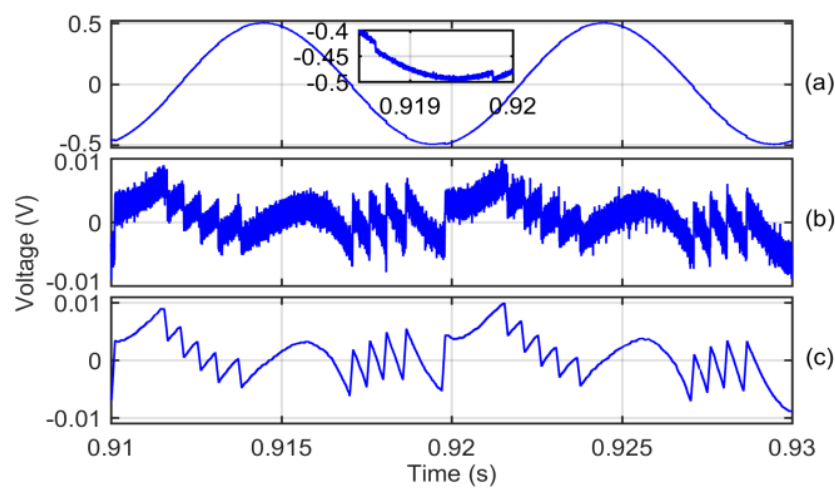

Fig. 13. (a) Experimental current-modulated laser output power signal for $f_{\mathrm{PZT}}=10 \mathrm{~Hz}$ with $150 \mathrm{~nm}$ amplitude. Modulating current signal had frequency of $100 \mathrm{~Hz}$, (b) recovered noisy SMI signal, and (c) RLS-ALE based filtered SMI signal.

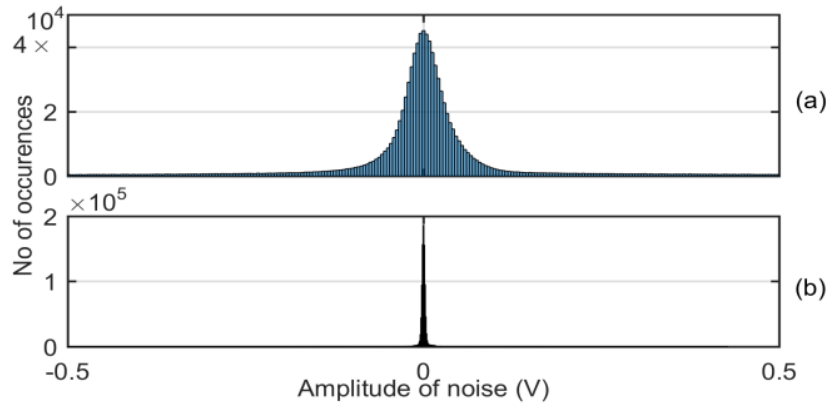

Fig. 14. Statistical distribution of RLS-ALE based recovered experimental noise: (a) Gaussian distribution of noise of SM signal of Fig. $13(\mathrm{~b})$ with standard deviation $(\sigma)$ of 0.28 and mean $(\mu)$ of 0.00084 , and (b) distribution with $\sigma=0.0439$ and $\mu=-0.00041$ for impulsive experimental noise of SM signal of Fig. 11 (a).

\section{CONCLUSION}

An adaptive solution of de-noising SMI signal is presented which requires neither separate noise-estimation nor filterparameter adjustments. The proposed algorithm effectively removes impulsive- and white-noise, and works wells for all major optical feedback regimes. The method is tested on both simulated- and experimental-SMI signals and the results are compared with standard LPF and DWT based filtering. This comparison shows that the proposed method provides better precision under all conditions without changing any key parameter. Noise reduction of speckle affected signals also demonstrates its robustness whereas the LPF and DWT 
excessively smoothed the SMI signal, causing false fringe detection. Various reported results indicate that the proposed method can be very useful for pre-processing of most SMI signals acquired under typical experimental conditions.

In addition, RLS-ALE also enables simple, direct, and accurate estimation of SNR of experimental SMI signals. Very good SNR estimations, over range of $0 \mathrm{~dB}$ to $40 \mathrm{~dB}$ approximately (see Table I) validate its usefulness for SMI, an area in which simple SNR estimators are needed.

\section{REFERENCES}

[1] S. Donati, "Developing self-mixing interferometry for instrumentation and measurements", Laser \& Photon. Rev., 6: 393417, May. 2012.

[2] T. Taimre, M. Nikolić, K. Bertling, Y. L. Lim, T. Bosch, and A. D. Rakić, "Laser feedback interferometry: a tutorial on the self-mixing effect for coherent sensing," Adv. Opt. Photon, vol. 7, pp. 570-631, 2015.

[3] Scalise, L.; Yanguang Yu; Giuliani, G.; Plantier, Guy; Bosch, T., "Selfmixing laser diode velocimetry: application to vibration and velocity measurement," Instrumentation and Measurement, IEEE Transactions on, vol.53, no.1, pp. 223-232, Feb. 2004.

[4] Zhao, Yunhe, et al, "Self-mixing fiber ring laser velocimeter with orthogonal-beam incident system." IEEE Photonics Journal 6.2, pp. 1-11, 2014.

[5] A. Ehtesham, U. Zabit, O. D. Bernal, G. Raja and T. Bosch, "Analysis and Implementation of a Direct Phase Unwrapping Method for Displacement Measurement Using Self-Mixing Interferometry," in IEEE Sensors Journal, vol. 17, no. 22, pp. 7425-7432, Nov. 2017.

[6] Magnani, A; Pesatori, A; Norgia, M., "Real-Time Self-Mixing Interferometer for Long Distances," Instrumentation and Measurement, IEEE Transactions on, vol.63, no.7, pp.1804-1809, July. 2014.

[7] U. Zabit, O. D. Bernal, S. Amin, M. F. Qureshi, A. H. Khawaja and T. Bosch, "Spectral Processing of Self-Mixing Interferometric Signal Phase for Improved Vibration Sensing Under Weak- and Moderate-Feedback Regime," IEEE Sensors Journal, vol. 19, no. 23, pp. 11151-11158, 2019.

[8] Ozdemir, S.K.; Ohno, I; Shinohara, S., "A Comparative Study for the Assessment on Blood Flow Measurement Using Self-Mixing Laser Speckle Interferometer," Instrumentation and Measurement, IEEE Transactions on, vol.57, no.2, pp. 355-363, Feb. 2008.

[9] S. Donati, and G. Martini, "3D Profilometry with a Self-Mixing Interferometer: Analysis of the Speckle Error." IEEE Photonics Technology Letters 31.7, pp. 545-548, 2019.

[10] Vogel, F.; Toulouse, B., "A low-cost medium-resolution range finder based on the self-mixing effect in a VCSEL," Instrumentation and Measurement, IEEE Transactions on, vol.54, no. 1, pp.428-431, 2005.

[11]Zhao, Yunkun, et al, "Temperature Measurement of the Laser Cavity Based on Multi-Longitudinal Mode Laser Self-Mixing Effect." IEEE Sensors Journal 19.12, pp. 4386-4392, 2019.

[12]Donati, S.; Norgia, M., "Self-Mixing Interferometry for Biomedical Signals Sensing," Selected Topics in Quantum Electronics, IEEE Journal of, vol.20, no.2, pp.104-111, 2014.

[13] Y. Wei et al., "Double-path acquisition of pulse wave transit time and heartbeat using self-mixing interferometry," Optics Communications, vol. 393, pp. 178-184, 2017.

[14]Gao, Yan \& Yu, Yanguang \& Xi, Jiangtao \& Guo, Qinghua \& Tong, Jun \& Tong, Sheng., "Removing the impulsive noise contained in a selfmixing interferometry system using outlier detection". Optical Engineering. 53. 124108, 2014.

[15]G. Giuliani and M. Norgia, "Laser diode linewidth measurement by means of self-mixing interferometry," in IEEE Photonics Technology Letters, vol. 12, no. 8, pp. 1028-1030, Aug. 2000.

[16] O. D. Bernal, H. C. Seat, U. Zabit, F. Surre, and T. Bosch, "Robust Detection of Non Regular Interferometric Fringes from a Self-Mixing Displacement Sensor using Bi-Wavelet Transform", IEEE Sensors Journal, vol. 16, No. 22, pp. 7910, 2016.

[17] A. A. Siddiqui, U. Zabit, O. D. Bernal, G. Raja, T. Bosch, "All Analog Processing of Speckle Affected Self-Mixing Interferometric Signals", IEEE Sensors Journal, vol. 17 , No. 18, pp. 5892-5899, 2017.

[18] Magnani, Alessandro, Alessandro Pesatori, and Michele Norgia, "Selfmixing vibrometer with real-time digital signal elaboration." Applied Optics 51.21, pp. 5318-5325, 2012.
[19] Arriaga, Antonio Luna, Francis Bony, and Thierry Bosch, "Speckleinsensitive fringe detection method based on Hilbert transform for selfmixing interferometry." Applied Optics 53.30, pp. 6954-6962, 2014.

[20]Y. Yu, J. Xi and J. F. Chicharo, "Improving the Performance in an Optical feedback Self-mixing Interferometry System using Digital Signal Pre-processing," 2007 IEEE International Symposium on Intelligent Signal Processing, pp. 1-6, 2007.

[21] Sun, Yuan \& Yu, Yanguang \& Xi, Jiangtao, "Wavelet Transform Based De-noising Method for Self Mixing Interferometry Signals". Proceedings of SPIE - The International Society for Optical Engineering. 8351. 15-. 10.1117/12.915940, 2012.

[22]Zhenghao Liu, Yanguang Yu, Yuanlong Fan, Jiangtao Xi, Qinghua Guo, and Jun Tong, "Eliminating influence of transient oscillations on a self-mixing interferometry," Optical Engineering 55(10), 104102, October. 2016

[23] Paulo S.R. Diniz, Adaptive Filtering Algorithms and Practical Implementation. New York, USA: Springer, 2008, ch. 5.

[24] Yanguang Yu, Tiemin Mei, Jiangtao Xi, Joe F. Chicharo, Huiying Ye, and Zhao Wang, "Eliminating noises contained in sensing signals from a self-mixing laser diode", Proc. SPIE 7656, 5th International Symposium on Advanced Optical Manufacturing and Testing Technologies: Optical Test and Measurement Technology and Equipment, 76560E, October. 2010.

[25]R. Kliese, Y. Lim, T. Bosch, and A. Rakić, "GaN laser self-mixing velocimeter for measuring slow flows," Optics Letters. 35, pp. 814-816, 2010.

[26] R. Matharu, J. Perchoux, R. Kliese, Y. Lim, and A. Rakić, "Maintaining maximum signal-to-noise ratio in uncooled vertical-cavity surfaceemitting laser-based self-mixing sensors," Optics Letters. 36, pp. 36903692, 2011.

[27]S. Donati, "Responsivity and Noise of Self-Mixing Photodetection Schemes," in IEEE Journal of Quantum Electronics, vol. 47, no. 11, pp. 1428-1433, Nov. 2011.

[28] Kim, Cholhyon, Cholman Lee, and O. Kwonhyok, "Effect of Linewidth Enhancement Factor on Fringe in a Self-Mixing Signal and Improved Estimation of Feedback Factor in Laser Diode." IEEE Access 7 pp. 28886-28893, 2019.

[29] G. A. Acket, D. Lenstra, A. Den Boef, and B. Verbeek, "The influence of feedback intensity on longitudinal mode properties and optical noise in index-guided semiconductor lasers," Quantum Electronics, IEEE Journal of 20, pp.1163-1169, 1984.

[30]T. Taimre and A. D. Rakic, "On the nature of Acket's characteristic parameter C in semiconductor lasers," Applied Optics 53, pp. 1001-1006, 2014.

[31]Bernal, O.D.; Zabit, U.; Bosch, T, "Classification of laser Self Mixing Interferometric signal under moderate feedback," Applied Optics, vol. 53, no. 4, pp. 702-708, 2014.

[32]C. Bes, G. Plantier and T. Bosch, "Displacement measurements using a self-mixing laser diode under moderate feedback", Instrumentation and Measurement, IEEE Transactions on, vol. 55, no.4, pp.1101-1105, Aug. 2006.

[33] Saeed V. Vaseghic, Advanced Digital Signal Processing and Noise Reduction. West Sussex, UK: John Wiley \& Sons Ltd, 2008, ch. 7.

[34]Peter M. Clarkson, Optimal and Adaptive Signal Processing. New York, USA: Routledge, 2017, ch. 5.

[35]G. Plantier, C. Bes, and T. Bosch, "Behavioral Model of a Self-Mixing Laser Diode Sensor," Quantum Electronics, IEEE Journal of 41, pp. 1157-1167, 2005.

[36] T. Hussain, S. Amin, U. Zabit, O. D. Bernal, T. Bosch. , "A High Performance Real-time Interferometry Sensor System Architecture", Microprocessors and Microsystems, Vol. 64, pp. 2333, Oct, 2018.

[37] O. D. Bernal, U. Zabit, F. Jayat and T. Bosch, "Sub- $\lambda / 2$ Displacement Sensor with Nanometric Precision based on Optical Feedback Interferometry used as a Non-Uniform Event-Based Sampling System," IEEE Sensors Journal, vol. 20, no. 10, pp. 5195-5203, 2020. 